\title{
Development of the CFD model to the high viscosity fluid mixing modeling
}

\author{
Roman Havryliv, Łukasz Radosiński, Maciej Szudarek, Sylwia Oleś \\ 1. Department of Chemical Engineering, Lviv Polytechnic National University, UKRAINE, Lviv, St.Yura square, 9, \\ E-mail: havrilivroman@gmail.com \\ 2. Novasell Ltd., POLAND, Wrocław, ul. Stablowicka 147, E-mail: lukasz.radosinski@ novasell.pl
}

3. Institute of Metrology and Biomedical Engineering, Warsaw University of Technology, POLAND, Warszawa, ul. Św. Andrzeja Boboli 8, E-mail: maciej.szudarek@pw.edu.pl

4. Department of Process Engineering and Chemical Technology, Gdańsk University of Technology, POLAND, Gdańsk, Gabriela Narutowicza 11/12, poczta.sylwia.oles@gmail.com

\begin{abstract}
The computational fluid dynamics (CFD) model has been developed in order to describe the mixing behavior of the liquid components and scale up the dissolver construction to industrial application. The Sliding Mesh approach has been selected for simulating rotating. For the numerical calculation of multiphase flow VOF multiphase model based on the Euler-Euler approach has been used.
\end{abstract}

Keywords - mixing, Non-Newtonian fluids, numerical simulation, free surface, Doughnut effect.

\section{Introduction}

Mechanical mixing processes are widely used in many industries such as chemical, pharmaceutical, food production, building materials production, etc. Among them, a special place is occupied by the high viscosity or non-Newtonian fluids mixing. These fluids have complex rheological properties, that create some difficult problems to provide an effective industrial mixing [1,2].

Fire-resistant paint production is a special case of liquid and solid components mixing. The industrial paint mixer machines are used to combine and disperse the solids pigments and fluid components. The best mixing results can be obtained when the geometry of the mixer vessel, the dissolver disc diameter, the velocity magnitude, and the height of the dissolver disc above the bottom of the vessel as well as the rheological liquid properties are matched to one another.

From the hydrodynamic point of view, the mixing efficiency can be determined by the Doughnut effect as a signal that the maximum mechanical power possible is being transferred into the liquid phase and furthermore that the liquid is being agitated so that all the agglomerates will eventually reach the dissolver disc [3].

Taking these aspects into account, testing and optimizing the mixing process with high viscosity fluids in industrial conditions is extremely expensive and time-consuming.

Recently, CFD simulations have been used to optimize and scale up the laboratory results. The CFD modeling techniques make it possible to evaluate the hydrodynamics of mixing, the efficiency of mixer design, mechanical power, efficiency, and determines the time necessary to reach the optimum dispersion result $[4,5]$.

In this regard, the development of an appropriate CFD model is a key task for the use of modern engineering approaches for high viscosity fluids mixing.

\section{Results and Discussion}

To develop and validate the CFD model the laboratory $10 \mathrm{~L}$ dissolver was used. The 3D model of the dissolver $(10 \mathrm{~L})$ is presented in Fig. 1a and fully complied with the laboratory installation. The dissolver disc diameter of $70 \mathrm{~mm}$ was located at the center of the vessel, and 97 $\mathrm{mm}$ from the bottom and was driven by a variable-speed motor that could operate in the range of 
50-3000 rpm. The height of the fluid in the vessel was set at $155 \mathrm{~mm}$. This is $57 \mathrm{~mm}$ above the dissolver disc.

The sliding mesh approach was selected to simulate the mixing process inside the computational domain. In accordance with this approach, the fluid domain was divided into two different domain zones (the moving located around the dissolver disc and stationary zones for the rest of the domain).

The unstructured polyhedral mesh was used for the meshing of the domain (Fig. 1b). To reduce the computational effort, only $1 / 16$ (one blade passage) of the overall domain was modeled and periodic conditions were applied to the boundaries. The final mesh was about 30181 elements.

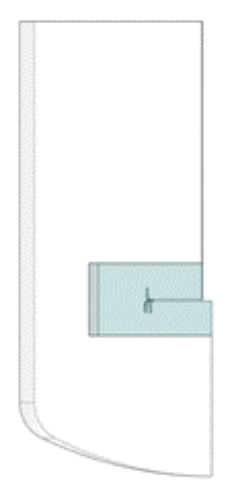

a)

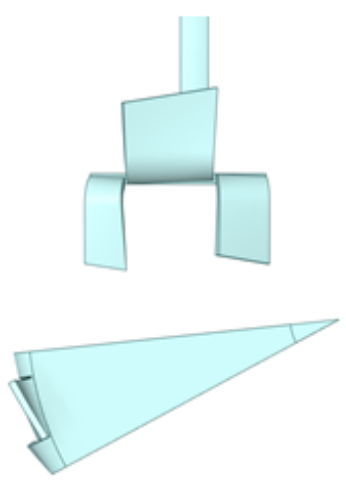

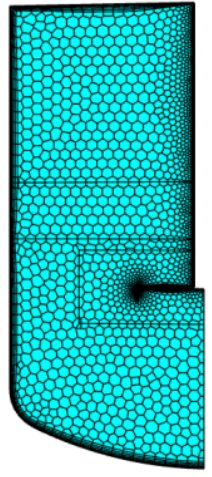

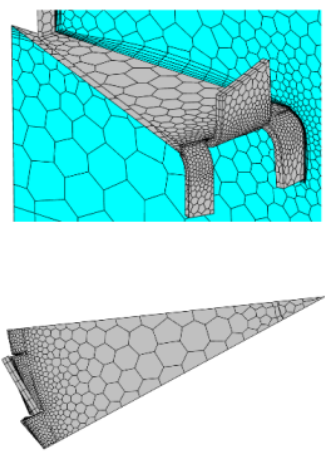

b)

Fig.1. Geometry and mesh.

ANSYS Fluent software version 2020R2 was used to simulate the fluid behavior inside the dissolver vessel. An Eulerian Multi-Fluid VOF multiphase model had to be used since a free surface between the fluids mixture (mill-base and let-down) and air existed.

To hydrodynamic modeling, in addition to governing equations (continuity and momentum), the Reynolds-Averaged Navier-Stokes (RANS) formulation has been used in the simulations to solve the flow field that requires an additional turbulence model for closure. In this study, the two-equation k- $\omega$ SST turbulence model was chosen to simulate the hydrodynamic performance in the mixing system.

No-slip wall condition was applied to the vessel wall, bottom, cover and to the shaft and the dissolver disc.

The computations were carried out with 24 parallel processors using a $2.6 \mathrm{GHz}$ Intel Xeon CPU E5-2690 v3 with 128 GB RAM. The simulation is converged when the residuals reach a plateau, as in the steady-state simulations.

In the first stage of the modeling, the mill-base and let-down mixture in 78/22\% vol. was used as a mixing highly viscous fluid. The mill-base has a non-Newtonian behavior and ranges from 550 to $1500 \mathrm{mPa} \cdot \mathrm{s}$ in shear condition. The rheological properties of the mixture, as well as the viscosity of each component, were determined by a rotational controlled stress rheometer (DV3TRV), equipped with a cone small sample adapter. The viscosity of the liquid mixture was in the range of 500 to $600 \mathrm{mPa} \cdot \mathrm{s}$.

Power consumption and velocity field are probably the most common approach to validate CFD models of stirred vessels. But in the case of the high viscous fluids mixing the hydrodynamic effect described before can be used as an additional tool.

CFD modeling results in Fig. 2 show the velocity field in vector forms Fig.2a and phases distribution profile Fig. $2 b$ in vertical cross-section for rotational frequency $n=3000 \mathrm{rpm}$. 


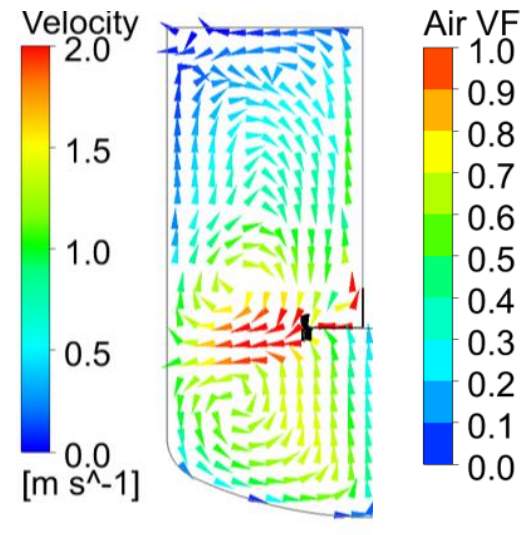

a

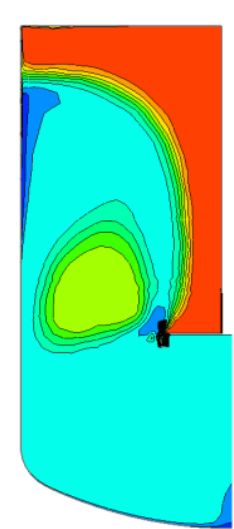

$\mathrm{b}$

Fig.2. CFD modeling results. (a) Velocity field vectors; (b) Contours of air volume fraction

The results show that the stream is divided into two parts. The one going downwards flows back to the middle of the dissolver disc along the bottom of the vessel and rises up to hit the disc once again. The second part flowing upwards has the same circular path, which is limited by the force of gravity and the rheological properties of the liquid mixture. As was noted before, the best dispersion results are obtained when the Doughnut flow pattern is maintained, which is visible in both figures.

\section{Conclusion}

The purpose of the study was to develop a CFD model to predict different features of the high viscous fluid mixing process. The CFD model included the mixing process, the sheardependent viscosity, and the free surface problem. The numerically predicted velocities field and flow with a free surface (the Doughnut effect) are show good agreement to the experimental measurements. The CFD also predicts the location and magnitude of the vortex formed due to the Doughnut effect and disk location. The proposed CFD model can be used to scale up the real mixing processes in industrial applications.

\section{Acknowledgments}

The work has been funded and realized within NCBiR European Funds project POIR.01.01.01-00-0218/19 ,Development of an innovative system for fire protection of steel structures."

\section{References}

[1] Arratia, P.E., Kukura, J., Lacombe, J., Muzzio, F.J., 2006. Mixing of shear-thinning fluids with yield stress in stirred tanks. AICHE J. 52, 2310-2322

[2] Zhu, H., Kim, Y., de Kee, D., 2005. Non-Newtonian fluids with a yield stress. J. NonNewtonian Fluid Mech. 129, 177-181

[3] Kazemzadeh, A., Ein-Mozaffari, F., Lohi, A., Pakzad, L., 2016. Effect of the rheological properties on the mixing of Herschel-Bulkley fluids with coaxial mixers: applications of tomography, CFD, and response surface methodology. Can. J. Chem. Eng. 9999, 1-13

[4] Cortada-Garcia, M., Dore, V., Mazzei, L., Angeli, P., 2017. Experimental and CFD studies of power consumption in the agitation of highly viscous shear thinning fluids. Chem. Eng. Res. Des. 119, 171-182

[5] Adams, L., Barigou, M., 2007. CFD analysis of caverns and pseudo caverns developed during mixing of non-Newtonian Fluids. Chem. Eng. Res. Des. 85, 598-604 